\title{
Servicio Cooperativo Interamericano de Salud Pública: Una historia de 20 años de colaboración con el Perú \author{
collaboration with Peru
} \\ Interamerican Cooperative Service of Public Health: A history of 20 years of
}

\author{
Carlos Bustios Romaní ${ }^{1, a}$, Martha Martina Chávez ${ }^{1, b}$, Juan Pablo Murillo ${ }^{1, c}$ \\ ${ }^{1}$ Departamento Académico de Medicina Preventiva y Salud Pública, Facultad de Medicina, Universidad Nacional Mayor de San Marcos. Lima, Perú. \\ ${ }^{a}$ ORCID: https://orcid.org/0000-0002-6958-6076 \\ ${ }^{b}$ ORCID: https://orcid.org/0000-0002-8410-9120 \\ ${ }^{\circ}$ ORCID: https://orcid.org/0000-0003-3710-2804
}

\section{An Fac med. 2019;80(4):515-27 / DOI: https://doi.org/10.15381/anales.v80i4.17254}

\section{Correspondencia: \\ Juan Pablo Murillo \\ jpmurillop@gmail.com}

Recibido: 6 de noviembre 2019

Aceptado: 27 de noviembre 2019

Publicación en línea: 28 de diciembre 2019

Conflictos de interés: Los autores

declaran no tener conflictos de interés.

Fuente de financiamiento: Autofinanciado

Citar como: Bustíos C, Martina M, Murillo JP. Servicio Cooperativo Interamericano de Salud Pública: Una historia de 20 años de colaboración con el Perú. An Fac med. 2019;80(4):515-27. DOI: https://doi. org/10.15381/anales.v80i4.17254

\section{Resumen}

Este artículo presenta información bibliográfica y testimonial sobre la historia del Servicio Cooperativo Interamericano de Salud Pública (SCISP) en el Perú durante el período 1942-1962, en el contexto de la aplicación de la "Política del Buen Vecino" del Gobierno de Estados Unidos en sus relaciones con América Latina. Los propósitos de este artículo son, primero, tratar sobre los alcances y limitaciones de los programas de tecnificación y modernización sanitaria que desarrolló el SCISP en nuestro país y, segundo, analizar la importancia de sus programas de adiestramiento y de capacitación en la constitución de una generación de sanitaristas que en el año 1962 definieron consensualmente los principios doctrinarios de una salud pública nacional, enraizada en la realidad local, que orientaron las intenciones de la política sanitaria oficial en las siguientes décadas.

Palabras clave: Salud Pública; Historia de la Medicina; Capacitación; Perú (fuente: DeCS BIREME).

\section{Abstract}

This article presents testimonial and bibliographic information about the history of the "Service Cooperative Inter-American of Public Health" (SCISP) in Peru during the period between 1942-1962, in the context of the implementation of the "Good Neighbor Policy" from the United States Government in its relations with Latin American. The first purpose of this article is to discuss the scope and limitations of technification and modernization public health programs which were developed by SCISP in our country; and second, discuss the importance of their training, and professional development programs in the development of a new generation of sanitarians who consensualmously defined in 1962, the doctrinal principles of the national public health, rooted in local realities, which it was influential in the official public health policy in the next decades.

Keywords: Public Health; History of Medicine; Training; Peru (source: MeSH NLM). 


\section{INTRODUCCIÓN}

Diversos estudios han relevado la importancia de las instituciones en el estado y en ese contexto la perspectiva histórica es una herramienta de enorme utilidad para entender los procesos de construcción del estado. En el Perú, las investigaciones en historia social de nuestras instituciones son relativamente recientes. En el caso del sector salud, existen pocos estudios que analicen los procesos de construcción institucional. Algunos autores han relevado el impacto de los determinantes externos en la construcción de instituciones. En el caso de América Latina, varios estudios han señalado la enorme influencia de la Política Exterior Norteamericana en el desarrollo institucional en salud en varios países latinoamericanos. En ese sentido, el Servicio Colaborativo Interamericano en Salud Pública fue una herramienta muy potente de la cooperación norteamericana en salud y su presencia tuvo un enorme impacto en el desarrollo de capacidades institucionales en la región. Así, el objetivo del presente artículo es describir las acciones del Servicio Colaborativo Interamericano de Salud Pública (SCISP) en el Perú y analizar su influencia en el proceso de construcción institucional del Ministerio de Salud del Perú en el periodo 1942-1962.

\section{La Segunda Guerra Mundial y la coope- ración en salud en América Latina}

Las nuevas condiciones mundiales económicas y comerciales, creadas por la segunda guerra mundial, llevaron al gobierno de Estados Unidos a organizar instituciones destinadas a instrumentar una nueva "Política del Buen Vecino" en sus relaciones con América Latina; además, existía el temor que las colectividades de residentes de origen alemán e italiano pudieran convertirse en focos de oposición a las políticas de defensa continental. Por ello, el gobierno de los Estados Unidos creó la "Oficina de Coordinación de Asuntos Interamericanos" (OCAI), por la orden ejecutiva $N^{\circ} 8840$ dictada por el Presidente Theodore Roosevelt, el 30 de julio de 1941; nombrándose a Nelson Rockefeller como el coordinador de la oficina. Al final del mismo año, la Oficina inició un Programa de Cooperación en Trabajo de Salubri- dad Pública, a cargo de la División de Salud y Saneamiento del "Instituto de Asuntos Interamericanos" (IAI), nueva dependencia de la OCAI. El IAI sería el antecedente de la "Agencia de los Estados Unidos para el Desarrollo Internacional" (USAID), creada veinte años después al inicio del gobierno de J. F. Kennedy ${ }^{(1,2,3)}$.

Los objetivos de la División de Salud y Saneamiento de la IAI fueron los siguientes:

- A nivel militar, mejorar las condiciones de salud, sobre todo en relación con las exigencias de las fuerzas armadas estadounidenses.

- Desde el punto de vista político, cumplir con los programas de salud y saneamiento asumidos de conformidad con la Resolución XXX de la Conferencia de Río de 1942.

- En los aspectos económicos, lograr un aumento de materiales esenciales para la guerra en zonas donde prevalecían malas condiciones de salud.

- En términos de relaciones exteriores, demostrar los beneficios tangibles de la democracia en marcha y ganar el apoyo de la población civil a la causa de los Estados Unidos frente a Alemania y sus países aliados.

Para cumplir con estos objetivos, la División de Salud y Saneamiento de la IAI debía establecer contratos o convenios de cooperación técnica y financiera con los gobiernos de los países latinoamericanos. Para lograr estos objetivos, los signatarios del Acta Final de la Tercera Conferencia de Ministros de Relaciones Exteriores de las Repúblicas Americanas, reunida en Río de Janeiro en enero de 1942, establecieron las bases para crear un Programa Cooperativo entre el IAI, como representante del gobierno de los Estados Unidos de América, y las demás repúblicas americanas. En la Resolución XXX de esta Conferencia, los gobiernos resolvieron cooperar entre sí para solucionar los problemas de salud pública, considerando medidas adecuadas para proteger la salud y las condiciones de salubridad, como una contribución esencial para la defensa continental. Entre ellas, la creación de programas cooperativos de asistencia técnica y financiera en zonas de interés estratégico mutuo ${ }^{(4)}$.
Posteriormente, en la Conferencia Sanitaria Panamericana de septiembre de 1942, uno de los temas centrales en la agenda fue la denominada: "Defensa Continental y Salud Pública". Al final del debate se acordó: "propiciar que se adopten las medidas emergentes en materia de cuarentena para armonizar la defensa de cada país con la defensa general del continente". La vigilancia y el control de las embarcaciones en tiempos de guerra requerían nuevos mecanismos de comunicación y de decisión que pasaban por el criterio de la conveniencia o el peligro militar, además de la tradicional prevención de las enfermedades transmisibles, lo que significó, una oportunidad para que los representantes de los países expresen, su adhesión a la defensa continental, también en el terreno de la salud pública ${ }^{(5)}$.

Para el mejor logro de sus objetivos, el Gobierno de los Estados Unidos suspendió la práctica de cooperación que había aplicado en la región hasta el inicio de la década de 1940; es decir, dejó de limitarse a proporcionar asesoría técnica, de servicios y de expertos a los países de la región de manera poco sistemática, para plantear un nuevo enfoque de colaboración, para el desarrollo a largo plazo de los llamados "servicios", con un sistema de propiedad y de administración conjuntos. En este sentido, el influjo norteamericano, que en un primer momento se efectuó mediante acciones del sector privado, se canalizó a través de instancias gubernamentales estadounidenses, conformando un vínculo más expedito de intereses con los países involucrados.

\section{INICIO Y PRIMEROS PROYECTOS DEL SERVICIO COOPERATIVO INTERAMERICANO DE SALUD PÚBLICA EN EL PERÚ: 1942-1960}

En el marco de aquellos acuerdos regionales, el Gobierno Peruano aprobó, por Resolución Suprema del 14 de julio de 1942 el convenio básico firmado tres días antes, por el ministro del Ministerio de Salud Pública y Asistencia Social (MSPAS), Dr. Constantino C. Carvallo, y el Brigadier General George C. Dunham, en representación del IAI y como direc- 
tor de la División de Salud y Saneamiento del mismo instituto. El convenio contemplaba la creación de un servicio técnico especial dentro del MSPAS, el "Servicio Cooperativo Interamericano de Salud Pública" (SCISP), que se financiaría con fondos del IAI y del Estado peruano, salvo los salarios de los miembros de la misión del IAl en el país que serían pagados directamente por el instituto. En el mismo convenio, se establecía que la cooperación estadounidense incluía la participación de especialistas de ese país, así como que la ayuda económica sería progresivamente decreciente - proporcionalmente - en los gastos de ejecución y mantenimiento de los programas de salud prioritarios. También se señalaba que el SCISP era un organismo autónomo, directamente subordinado al MSPAS, a cargo de un Director Médico funcionario del IAI y de nacionalidad estadounidense. El primer director del SCISP fue el Dr. Edward A. Westphal ${ }^{(6)}$.

Desde el inicio de sus operaciones en el país, el SCISP dispuso de la autonomía administrativa y de los recursos humanos y financieros necesarios, para garantizar el desarrollo apropiado de sus acciones programadas. Para ello, se constituyó como una organización vertical dirigida y operada por profesionales y técnicos estadounidenses, que progresivamente serían reemplazados por profesionales o técnicos peruanos adiestrados en el SCISP y, luego, capacitados en escuelas de salud pública de universidades de EE.UU. o de otros países de la región. Los estándares técnicos exigidos y las condiciones de trabajo del personal del SCISP fueron siempre superiores a los existentes en otras dependencias administradas por el MSPAS, tal como lo relata el testimonio del Dr. Manuel Villacrespo: "En 1946, inicié mi carrera profesional como médico contratado en el SCISP (...). En el nivel operativo, el SCISP actuaba con una completa autonomía del resto del ministerio, incluso tenía una escala remunerativa diferente para su personal. En 1946, el sueldo mensual del Director General del Ministerio era de 800 soles y en el caso de un médico sanitario del nivel local alcanzaba de 200 a 400 soles; en cambio, a un médico recién egresado que trabajaba en el SCISP recibía un sueldo de 1,200 soles para ir a lquitos" (7).
El programa acordado inicialmente incluía tres grandes proyectos: Saneamiento de la Cuenca Amazónica, Saneamiento del Puerto de Chimbote y Campaña de Medicina Preventiva. De esta manera, el SCISP atendía los intereses estratégicos prioritarios, en esos años, de los gobiernos de los Estados Unidos y del Perú. Sus acciones en la selva amazónica y en la costa norte atendían los intereses de ambos países; los bélicos de Estados Unidos - mayor producción de materias primas (quina, jebe y otras) y la posibilidad de establecer bases aeronavales para la defensa continental -; y atendían a las limitaciones los fiscales del Perú, en tanto que los recursos locales, siempre limitados, nunca habían atendido las demandas de servicios de salud o de otra índole de la población asentada en esos lugares ${ }^{(8)}$.

\section{El Saneamiento de la Cuenca Amazónica}

Las demandas internacionales derivadas de la II Guerra Mundial revalorizaron la importancia estratégica y económica de la Amazonía peruana $y$, en consecuencia, de la colonización interna para su desarrollo. Existió un renovado interés de parte del gobierno, del ejército, los empresarios y las órdenes religiosas por la modernización de la selva, así como por impedir la difusión de la lepra. Esta revalorización se expresó en el campo de la salud pública en el especial apoyo que el MSPAS, especialmente a través de la participación del SCISP, comenzó a prestar a los servicios de salud ubicados en la Amazonía.

Esa participación protagónica del SCISP consistió en el financiamiento, construcción, gestión y operación de una nueva red de establecimientos o unidades de producción de servicios con diferentes niveles de complejidad, que estaría a cargo de la campaña de saneamiento de la cuenca. Las características de la red obedecían a la necesidad de responder a las exigencias generadas por las grandes dimensiones de la cuenca amazónica, las dificultades geográficas y de dispersión demográfica de la población, así como la precariedad de las carreteras de penetración.

De acuerdo a la racionalidad sanitaria estadounidense utilizada por el IAI en sus proyectos, las actividades de la campaña se efectuaban, de manera complementaria, en tres áreas operativas distintas: asistencia médica-hospitalaria, campaña de medicina preventiva, y educación sanitaria. La primera área operativa, incluía a los hospitales, dispensarios policlínicos, lanchas sanitarias y dispensarios móviles, que atendian las necesidades de los pobladores que vivían a lo largo de la carretera Tingo María-Pucallpa. La segunda área incluía a los puestos sanitarios y puestos de distribución de atebrina, ubicados en aquellos lugares donde no era práctico construir un dispensario; así como los centros de salud preventiva de Iquitos, Moyobamba y Yurimaguas ${ }^{(6,8)}$.

La forma en que se desarrollaban las actividades operativas del SCISP en la Amazonía es descrita por dos distinguidos sanitaristas peruanos que iniciaron su carrera en este servicio. Sanitaristas que, posteriormente, ocuparían altos cargos en organismos nacionales e internacionales de salud pública:

"Los primeros años de mi carrera profesional transcurrieron en el SCISP, como uno de los médicos del hospitalito de Contamana, donde la gente concurría con toda su familia. Los shipibos y las tribus cercanas acampaban alrededor del local, y después de su atención regresaban a sus tierras. Pienso que se estaba creando una conciencia ciudadana de la importancia de estas actividades en la población indígena a pesar de la necesidad de buscar intérpretes para comunicase con los médicos. Uno de los problemas era la transferencia de casos al hospital de lquitos (...). Nuestro trabajo en el hospitalito se complementaba con nuestra participación en el Servicio Sanitario Fluvial, que hacía viajes por el Ucayali. Marañón y Huallaga. El viaje en una lancha sanitaria - con médicos, enfermera y sanitarios entre lquitos y Pucallpa duraba de 20 y 25 días y otro tanto de regreso. Sin embargo, los pobladores ribereños sabían más o menos la fecha en que pasaba la lancha e iban para ser atendidos, no solamente la población del puerto sino la de los caseríos vecinos. Además, en los puestos fijos, se procedía a la vacunación y se hacian campañas de educación sanitaria..." (7). 
"En 1949, inicié mi carrera profesional como médico en el SCISP, mi sede era el Hospital-Dispensario № 1 en Caballococha. Ahi, realizaba acciones preventivopromocionales y asistenciales; además, tenía que hacer viajes en un bote deslizador, con un sanitario, para cumplir con el Servicio Sanitario Fluvial que incluía acciones vinculadas con la atención de los enfermos que nos esperaban a lo largo de los ríos; la aplicación de medidas preventivas, especialmente inmunizaciones; la atención del parto; extracciones dentarias; $y$, la transferencia de pacientes que requerían una atención de mayor complejidad. Aunque, en épocas de sequía de los ríos no entraban lanchas, y el único medio de transporte era el hidroavión que hacía servicio una vez por semana, así como el único medio de comunicación con el exterior era la radio de la Corporación de Amazonas (...) En 1950 hubo una epidemia de viruela en las provincias de Moyobamba y Rioja de San Martin. Había alrededor de 140 casos de viruela. Nuestro trabajo consistió en instalar puestos para la cuarentena, internar a los enfermos en salas especiales de aislamiento y a vacunar a toda la población de la zona. Estuvimos trabajando en la campaña cerca de cinco meses y alcanzamos coberturas útiles de vacunación. Se alcanzó así el control del brote, con una letalidad mínima, debido que el SCISP estaba muy bien organizado y nos suministraban todas las medicinas y los apoyos que se requerían" ${ }^{(9)}$.

En el momento de su más alto desarrollo, el SCISP llegó a instalar en estas zonas ocho importantes centros de operaciones. Así, por ejemplo, el centro de salud del SCISP en Tingo María proporcionaba más servicios de salud pública que cualquier otro en el Perú, aparte de Lima. El centro ofrecía educación en salud, control de enfermedades infecciosas, atención a cargo de parteras, higiene dental, control de vectores y roedores, abastecimiento de agua, eliminación de aguas residuales e ingeniería sanitaria. El SCISP también tenía un hospital en la ciudad que atendía casos de cirugía tradicional y servicios médicos, incluyendo maternidad y cuidado pediátrico, así como tratamiento para la tuberculosis. Los residentes en zonas rurales tenían acceso a centros de salud, postas de salud y a servicios rurales ambulatorios ${ }^{(10)}$.
Al concluir sus actividades en el país, autoridades del SCISP destacaron que durante su gestión en la Amazonía se habían construido: el Hospital de Iquitos (80 camas); los de Yurimaguas, Pucallpa, Tingo María y Tarapoto, cada uno de ellos con 40 camas; así como los dispensariohospitales de Caballococha y de Contamana, cada uno con 15 camas. Los siete establecimientos poseían una construcción de madera adaptada a las condiciones climatéricas y topográficas de la región. También destacaban la adquisición de lanchas sanitarias para el servicio fluvial, que después de ser dotadas de personal, equipo y recursos sanitarios, recorrían los diferentes ríos y atendían a las poblaciones ribereñas dispersas ${ }^{(10)}$. Además, dichos establecimientos se convirtieron en centros para el entrenamiento de enfermeras e inspectores sanitarios de salud pública, desde donde se facilitaba la salida de peruanos a los Estados Unidos para el estudio de esta materia.

Las condiciones de la organización y la disponibilidad de recursos de las unidades sanitarias departamentales administradas por el SCISP, así como de la integración de sus acciones contrastaban con las existentes en otras unidades sanitarias administradas directamente por el MSPAS. Un distinguido sanitarista peruano, Alfredo Lynch haría el siguiente comentario sobre dicho contraste, tomando como ejemplo la situación de la Unidad Sanitaria Departamental de Ica en el año 1945: "La organización sanitaria en el departamento de lca se reducía a un médico sanitario departamental y un médico sanitario en cada una de las provincias, además de igual número de obstetrices titulares y unos cuantos vacunadores quienes no habian tenido ninguna preparación para el cargo. Las actividades desarrolladas por el servicio sanitario eran casi todas de atención a enfermos indigentes, acciones de carácter médico legal y algunas vacunaciones contra la viruela (...). Existian también consultorios materno-infantiles, antivenéreos y antituberculosos, los cuales no dependían de la Unidad Sanitaria Departamental de Ica, sino de los servicios nacionales dirigidos desde el nivel central del Ministerio de Salud. Cada uno de esos consultorios tenía su plan y administración separada sin coordinación entre ellos; además, eran muy celosos en defender su autonomía y como rara vez recibían la visita de los departamentos técnicos de los servicios nacionales del Ministerio de Salud (...) faltaba la supervisión y orientación adecuada. Esta era en líneas muy generales la situación de los servicios públicos de salud a principios de 1945. La excepción era el SCISP creado en Julio de 1942" (11).

\section{Saneamiento del Puerto de Chimbote}

Otra labor tempranamente encomendada al SCISP fue el saneamiento de Chimbote, pequeña ciudad portuaria ubicada en la costa norte del país, que según el censo de 1940 tenía 4500 habitantes. La prioridad otorgada al proyecto partía del hecho que el gobierno peruano había decidido instalar en esa parte del país las bases de una industria siderúrgica nacional considerada, entonces, esencial para el desarrollo del país. Sin embargo, la ciudad carecía de los más elementales servicios de agua potable y alcantarillado, así como estaba rodeada de pantanos que contribuían a mantener elevados índices de morbimortalidad por malaria. Situación epidemiológica que impedía la ejecución de obras planeadas con aquella finalidad industrial. Además, la configuración de la bahía de Chimbote y su posición geográfica se adecuaban a la instalación de una base naval en el Pacífico para fines de defensa continental ${ }^{(6,8)}$.

El Proyecto del Saneamiento de Chimbote incluía las siguientes obras: la desecación y drenaje de siete pantanos que ocupaban 887000 metros cuadrados, la instalación de un sistema de agua potable y de desagüe; así como la construcción de un moderno hospital de 40 camas y un servicio ambulatorio. Obras que el SCISP inició con un fuerte aporte financiero en el año 1943 acompañadas de una campaña para la prevención y tratamiento de la malaria ${ }^{(6,8)}$.

Para participar en la evaluación de la construcción del hospital, los servicios de agua y alcantarillado, desecación de los pantanos, así como iniciar las acciones de control de la malaria el SCISP contrató al sanitarista peruano Carlos Quiroz Salinas, quien posteriormente sería el primer director del nuevo Hospital "La Caleta de Chimbote" inaugurado en mayo de 1945. 
Quiroz, quien permaneció en este cargo hasta el año 1948, nos informa: "Las actividades del hospital no se limitaron a la labor asistencial, sino, también a programas de prevención, mediante el establecimiento -dentro del hospital- de los consultorios: materno infantiles, escolar, enfermedades de transmisión sexual, etc. Además, el municipio cedió unos ambientes en su local para el trabajo de los inspectores sanitarios y tres auxiliares de enfermería, a las que se les preparó para el trabajo de visitas domiciliarias, programa que, posteriormente, se reforzó con la llegada de una enfermera de salud pública y una educadora para la salud. Luego de la llegada de dos cirujanos se amplió la labor asistencial (...). Los accidentes ocurrían con cierta frecuencia, debido al incremento de las obras del puerto, de la siderúrgica, así como al tráfico de la carretera panamericana" (12).

Por otro lado, en la década de los cuarenta aún se desconocía el potencial industrial de la anchoveta que convertiría a Chimbote en el primer puerto pesquero del mundo. Cuatro décadas después, el mismo Quiroz hacía los siguientes comentarios sobre los resultados finales del proyecto: “... la falta de interés del gobierno norteamericano una vez terminada la guerra en 1945, dejo trunca todas las obras que había emprendido con el objeto de hacer de Chimbote una ciudad dotada de todos los servicios básicos de saneamiento (agua, desagüe, desecación de lagunas)... Afortunadamente con el advenimiento del DDT en1946, se realizó en Chimbote uno de los primeros ensayos en el Perú con lo que la malaria desapareció" (12).

En 1955, adicionalmente a sus otros compromisos, el SCISP asumió la responsabilidad de la reorganización, dirección y gestión integrada de todas las actividades sanitarias en el departamento de La Libertad. De acuerdo a los términos de la extensión del convenio, el SCISP procedió a capacitar el personal de salud correspondiente, así como a integrar los diversos servicios de salud pública del departamento, dentro de una unidad de salud debidamente organizada ${ }^{(13)}$.

\section{Campaña de Medicina Preventiva}

Edward A. Westphal, primer director del SCISP en el Perú, pronunció un discurso con ocasión de la inauguración del Centro de Medicina Preventiva del Rímac (1945) donde resumía las ideas básicas de la doctrina integracionista de las funciones preventivas que orientaba el convenio entre los gobiernos de EE UU y del Perú: "La complejidad y multiplicidad de las funciones preventivas en el campo de la Salud Pública, cuyo desempeño es responsabilidad que recae integramente en el Estado, ya no permite la dispersión de esfuerzos y duplicación del presupuesto económico, cuando estas se realizan por entidades separadas como son los diferentes dispensarios, los centros de asistencia materno-infantil, los puestos de vacunación, etc. Cada uno de ellos encara la correspondiente faceta de los problemas sanitarios. Pero es un hecho reconocido que los diversos aspectos de estos problemas están íntimamente ligados unos con otros y guardan relaciones de dependencia mutua. El Centro de Medicina Preventiva trata de lo prevenible, el tratamiento de los casos de enfermedades transmisibles, en cuanto ellos constituyen un riesgo para la salud de la comunidad, el control higiénico de los tres períodos del proceso maternológico; la Higiene Infantil, Pre-escolar y Escolar; la Higiene Dental; la Educación Sanitaria de la población; y la promoción del mejoramiento del saneamiento ambiental. Del Centro de Medicina Preventiva, estas labores serán irradiadas al ambiente de la familia... por medio de las visitas domiciliarias a cargo de enfermeras que llevaran a cabo la educación individual que es el aspecto más interesante de la campaña de educación sanitaria. Este centro de medicina preventiva, constituye un puesto de avanzada y paradigma de lo que debe ser la futura sanidad nacional" (14).

El Centro de Medicina Preventiva del Rímac, construido por el SCISP e inaugurado en julio de 1945, comenzó sus actividades como un "modelo de atención preventiva" que debía ser aplicado en nuestro país. Para ello se contó con el aporte de consultores estadounidenses $y$, progresivamente, de profesionales peruanos que regresaban al país después de haber sido capacitados en escuelas de salud pública de Estados Unidos y Puerto Rico. El director fundador del centro fue Mario León Ugarte, que regresaba al país después de obtener el grado de maestría en salud pública en la Universidad Johns Hopkins. En la ceremonia de inauguración estuvieron presentes: el Presidente de la República, Manuel Prado; el Ministro de Salud, Constantino Carvallo y el director del SCISP (15).

Siguiendo el modelo de Centro de Salud norteamericano se consideraron, en el primer Plan de Organización del Centro de Medicina Preventiva del Rímac, tres tipos de actividades sanitarias centradas en el paciente ${ }^{(15)}$ :

- Actividades generales: (i) investigación de la condición social del paciente; (ii) orientación de los pacientes que requieren atención asistencial; (iii) cursos de entrenamiento en medicina preventiva para estudiantes de medicina, enfermeras visitadoras y personal auxiliar que servirá en el Centro.

- Medicina preventiva general: (i) exámenes médicos periódicos para los indigentes; (ii) asistencia dental a indigentes; (iii) distribución de leche para madres indigentes. A través del programa de higiene $y$ de medicina preventiva.

- Control de enfermedades contagiosas: (i) diagnóstico de las enfermedades contagiosas (sífilis, gonorrea, chancro, TBC, parasitismo intestinal y malaria); (ii) tratamiento de los enfermos indigentes comprendidos en el grupo anterior de enfermedades; (iii) localización de los contactos de las enfermedades infecto-contagiosas; (iv) acciones de laboratorio correspondientes; ( $v$ ) inmunización contra las demás enfermedades infecto-contagiosas (viruela, difteria, coqueluche, tifoidea, tétanos, fiebre amarilla); (vi) divulgación sanitaria (cartillas, afiches e instrucción verbal).

El Centro de Medicina Preventiva del Rímac, se constituyó en el más importante centro de cuidado de salud preventiva para la ciudad de Lima, ampliando sus actividades de salud pública para incorporar la atención de higiene en todas las edades, control de TBC, salud sexual, 
higiene dental, nutrición, ingeniería sanitaria y estadística. Los éxitos del SCISP con sus servicios preventivos conducían a establecer excelentes relaciones con el MINSA, que recurría al SCISP para la asistencia en educación en salud, nutrición y estadística en salud. También se convirtió en un centro para el entrenamiento en salud pública de enfermeras e inspectores sanitarios y posibilitó el acceso de muchos peruanos a los Estados Unidos para seguir estudios en salud pública.

\section{OTROS PROGRAMAS DEL SERVICIO COOPERATIVO INTERAMERICANO DE SALUD PÚBLICA EN EL PERÚ: 1942-1960}

\section{Capacitación del personal de salud}

Los programas del SCISP no se limitaron a enfrentar problemas técnicos operativos de cuidado de la salud, sino que se preocuparon en capacitar al personal nacional para que tomara paulatinamente a su cargo la responsabilidad de conducir los programas y proyectos de salud. De esta manera, cientos de profesionales realizaron ciclos y cursos de orientación en los servicios operados por el SCISP. Además, sus autoridades prestaron especial atención a su programa de becas y entrenamiento de personal en universidades de Estados Unidos, Brasil, Chile y México ${ }^{(10)}$.

Además, la decisión del SCISP de contratar personal local en medicina e ingeniería para las nuevas instalaciones médicas contribuyó a crear empleo y entrenamiento calificado para un número importante de profesionales y técnicos. Asimismo, sentó las bases para que, en los años siguientes, la cooperación norteamericana promoviera una política sostenida de brindar entrenamiento en los Estados Unidos a los profesionales peruanos de la salud. Esto constituyó un factor clave para el éxito de las mejoras en muchos aspectos de la salud pública peruana. Entre los años 1942 y 1960 más de 150 profesionales peruanos adquirieron capacitación especializada en universidades y escuelas de salud pública de Estados Unidos y del continente, utilizando becas concedidas por el IAI. La distribución de las becas fue la siguien- te: salud pública: 44; servicio social: 16; ingeniería sanitaria: 14; enfermería: 9; nutrición y dietética: 6; administración hospitalaria: 5; educación sanitaria: 4; veterinaria: 3 ; entre otras especialidades y ramas técnicas.

La mayoría de esos becarios ocuparon, al regresar al Perú, posiciones dirigentes en el SCISP o el MSPAS, siendo muy importante su participación en la profesionalización y la tecnificación de las decisiones políticas, administrativas y operativas en el campo de la salud pública nacional. Es así, que, cinco de ellos ocuparon el alto cargo de director general de salud del MSPAS (equivalente al cargo actual de viceministro del Ministerio de Salud), entre los años 1942-1969: César Gordillo Zuleta (1942-1945), Julio Muñoz Pugliesevich (1957-1959), Jorge Atkins Morales (1959-1960), Carlos Quiroz Salinas (1962-1967) y Manuel Villa Crespo (1967-1969).

Además, el Centro de Salud de Medicina Preventiva del Rímac se convirtió muy pronto, con el apoyo técnico y financiero del SCISP, en el primer centro de entrenamiento práctico para personal de salud pública del país. Para este propósito se contó con el aporte de consultores norteamericanos y profesionales peruanos recién entrenados en salud pública en el extranjero, becados por el SCISP. En el año 1945, bajo la dirección de una enfermera de salud pública norteamericana, se inició el primer Curso de Enfermería en Salud Pública. Desde esa fecha hasta el año 1962 egresaron once promociones con un total de 160 enfermeras de salud pública. Además, la escasez de personal profesional de enfermería obligó al SCISP a iniciar la preparación formal de personal auxiliar, estableciendo cursos de entrenamiento para visitadoras de higiene y auxiliares de enfermería en el campo hospitalario y sanitario, según las necesidades ${ }^{(15)}$.

\section{Saneamiento ambiental}

La importancia que el SCISP concedió a los programas de saneamiento ambiental siempre fue significativa. Lo que se expresó, no solamente con las obras de Chimbote y las campañas contra las enfermedades transmisibles, sino con la construcción de pequeños sistemas de agua potable y la construcción y distribución de letrinas fabricadas en los talleres de las Unidades Sanitarias que el SCISP administraba en el país: La Libertad, Rímac, Santa, Tingo María y San Martín. Asimismo, por el inicio de la formación de técnicos de saneamiento en dichos talleres que luego se encargarían de dirigir esas obras en otras partes del país. Obras que pronto, con la aparición de las barriadas en las grandes ciudades, se convertirían en una práctica rutinaria en las Unidades Sanitarias del MSPAS ${ }^{(13)}$.

Importancia que también se manifestaba en la estructura central del SCISP donde se distinguía la División de Ingeniería Sanitaria, creada para dar unidad de comando y supervisar las labores de campo llevados a cabo por las estructuras de ingeniería sanitaria de dichas Unidades. En el año 1956 el Programa de Ingeniería Sanitaria del SCISP tenía un Jefe de Programa, un Jefe de Sección de Estudios de Proyectos, y, un Jefe de Sección de Saneamiento. Además, en cada una de las unidades sanitarias que administraba el SCISP tenía una sección local, a cargo de un ingeniero y de varios inspectores sanitarios. En total trabajaban en el Programa 11 ingenieros sanitarios o civiles y 34 inspectores sanitarios ${ }^{(16)}$.

\section{Administración de servicios especiales del Ministerio de Salud Pública y Asisten- cia Social \\ Departamento de Higiene Industrial e Instituto de Salud Ocupacional: 1948-1960}

Desde abril de 1948, en virtud del Acuerdo № 40 que suscribieron los gobiernos del Perú y de EE.UU., el SCISP asumió la administración del Departamento de Higiene Industrial del MSPAS. Profesionales con reconocimiento mundial como el Dr. Vintinner y el Ing. Bloomfield trabajaron varios años en el Departamento de Higiene Industrial, luego, Instituto de Salud Ocupacional (ISO) del Perú ${ }^{(17)}$.

Con los aportes del SCISP y los de la Ley 10833 (contribución del 1,8\% del valor total de las planillas de empresas mineras y conexas), el Departamento de Higiene Industrial pudo convocar $y$, luego, capacitar a personal profesional peruano en universidades de EE.UU., así 
como equiparse de manera suficiente para desarrollar actividades de alto nivel tecnológico para la época. Tanto en los campos de la medicina e ingeniería ocupacional y ambiental, del laboratorio clínico y químico toxicológico. En abril de 1949, el departamento inició sus actividades de investigación epidemiológica en los asientos mineros del centro del país.

En 1957, el departamento es cambiado de denominación por el del "Instituto de Salud Ocupacional" (ISO). Ese mismo año se construyó, con fondos propios, su local institucional ubicado en el distrito de Lince, y el SCIPS lo dota de modernos equipos para el cumplimiento de sus funciones. El ISO fue el primero de su campo en crearse en la región andina $y$, en reconocimiento de su desarrollo, entre 1948 y 1962, se convirtió en el centro de capacitación en salud ocupacional para la región. Los países vecinos empezaron a usar los programas del ISO para fines de entrenamiento ${ }^{(17)}$.

Al retirarse del país, el SCISP transfirió la administración del ISO al MSPAS. Poco después, el gobierno peruano promulgó un dispositivo legal por el que se dejaba sin efecto la Ley 10833 que proveía fondos al ISO como contribución de las empresas mineras. Este hecho fiscal y otros de carácter administrativo provocaron la disminución de las actividades del ISO y el retiro del personal profesional calificado.

Departamento de Nutrición e Instituto de Nutrición: 1949-1960

En 1949 el MSPAS firmó un convenio adicional con el IAI para reequipar y organizar el Departamento de Nutrición del MSPAS, que comenzó a ser operado como un Programa del SCISP, bajo la dirección del Dr. Carlos Collazos. La disponibilidad de modernos laboratorios permitió al programa realizar investigaciones sobre el valor nutritivo de los alimentos y los aspectos bioquímicos de la nutrición humana. Es así, que en el año1951 el departamento inició en el país encuestas sobre alimentación y el estado nutritivo de las poblaciones de las tres regiones naturales del país, con la asistencia técnica de expertos en estadística del Servicio de Salud Pública de EE.UU. Los primeros resultados de tales estudios fueron socia- lizados por el departamento, que comenzó a formular recomendaciones dietéticas, desarrollar actividades educativas y resolver consultas vinculadas con los problemas de la especialidad. Ese mismo año el Departamento de Nutrición identificó en su estructura administrativa la Sección de Profilaxia del Bocio ${ }^{(18,19)}$.

En el año 1957, el departamento se convirtió en el Instituto de Nutrición del MSPAS, aunque su programa de actividades siguió bajo la administración técnica del SCISP, siempre a cargo del Dr. Collazos. En marzo de 1960, el Instituto publicó el libro La Alimentación y el Estado de Nutrición en el Perú; cuyo contenido era el resultado de ocho años de investigaciones sobre los patrones dietéticos en la costa, la sierra y la selva del Perú. El estudio se realizó con la participación de la Universidad de Harvard, tanto en los aspectos técnico-consultivos como en la organización y ejecución de los trabajos ${ }^{(20)}$. En 1961, se firmaron convenios con la Universidad de Harvard para la capacitación de profesionales peruanos, así como para recibir asistencia técnica de esta institución.

Instituto Nacional de Higiene y Salud Pública e Instituto Nacional de Salud: 1955-1960

A principios del año 1955, de acuerdo con un convenio firmado por el Ministro de Salud Pública y por el representante del IAI, subsiguientemente aprobado por Resolución Suprema del 31 de diciembre de 1954, el SCISP asumió la administración del "Instituto de Higiene y Salud Pública” del MSPAS - "Instituto Nacional de Salud" (INS), desde 1957- que se ocupaba de la producción de vacunas y otros productos biológicos. "Luego de realizar una reorganización total de la estructura administrativa del laboratorio, el SCISP creó un sistema de producción de primer nivel. Al cabo de seis años, bajo la administración del SCISP, se logró despertar la confianza en las vacunas peruanas y se pudo reemplazar las vacunas importadas para enfermedades tales como sarampión, tos convulsiva, tétano y rabia con productos locales; por primera vez se inició su exportación" (21).

La producción de vacunas fue uno de los logros más importantes de las acciones de transferencia tecnológica del
SCISP. Se produjeron vacunas para la tos convulsiva, el tétanos y la rabia; sin embargo, el logro más significativo fue la producción de una vacuna para la viruela de gran calidad, la cual incluso se exporto a otros países, como Pakistán, India, Brasil, Bolivia, Uruguay entre otros ${ }^{(40)}$ (Figura 1).

División de Educación para la Salud: 1952-1960

Por otro lado, la educación eanitaria, como un servicio técnico del MSPAS, se inició en el Perú en el año 1952, encargándose al SCISP la organización y el desarrollo de un programa nacional. En coordinación con la División de Educación para la Salud del Ministerio, el SCISP desarrolló tres cursos de formación de educadores de salud pública (en los años 1952, 1956 y 1960), de los cuales egresaron un total de 22 educadores. De este total, 16 trabajan en el MSPAS en el año 1964. Además, el SCISP envió a seis de ellos a escuelas de salud pública del extranjero ${ }^{(22)}$. Si bien existió una gran voluntad política para el desarrollo del programa, existen evidencias de importantes limitaciones en su operación. El trabajo de campo de los educadores sanitarios, mostraron que las acciones del programa generaban un conjunto de expectativas para el desarrollo de intervenciones sobre las condiciones ambientales insalubres a nivel local, las cuales muchas veces, ante la falta de recursos, se llevaban a cabo a partir de iniciativas impulsadas por los educadores en alianza con las comunidades $u$ otros actores sociales ${ }^{(23)}$. En la tabla 1 se muestra una relación de los más importantes funcionarios del Ministerio de Salud que recibieron formación en el extranjero financiada por el SCISP.

El Servicio Colaborativo Interamericano de Salud Pública alcanzó su máximo nivel de despliegue en el año 1954. Contaba con tres divisiones, una de servicios locales de salud que incluía las unidades de salud del Rímac, Tingo María, San Martín, La Libertad y la Posta de Satipo. En 1957 el SCISP mantenía en operación 7 hospitales generales, que había construido o rehabilitado, un hospital de maternidad, un centro de cuidado infantil, un preventorio de tuberculosis, 8 centros de salud y 35 postas médicas, además de contar con 9 lanchas dispensario, para brindar atención sanitaria a los ríos de la selva. Las unidades territoriales, eran en la práctica, pequeños 


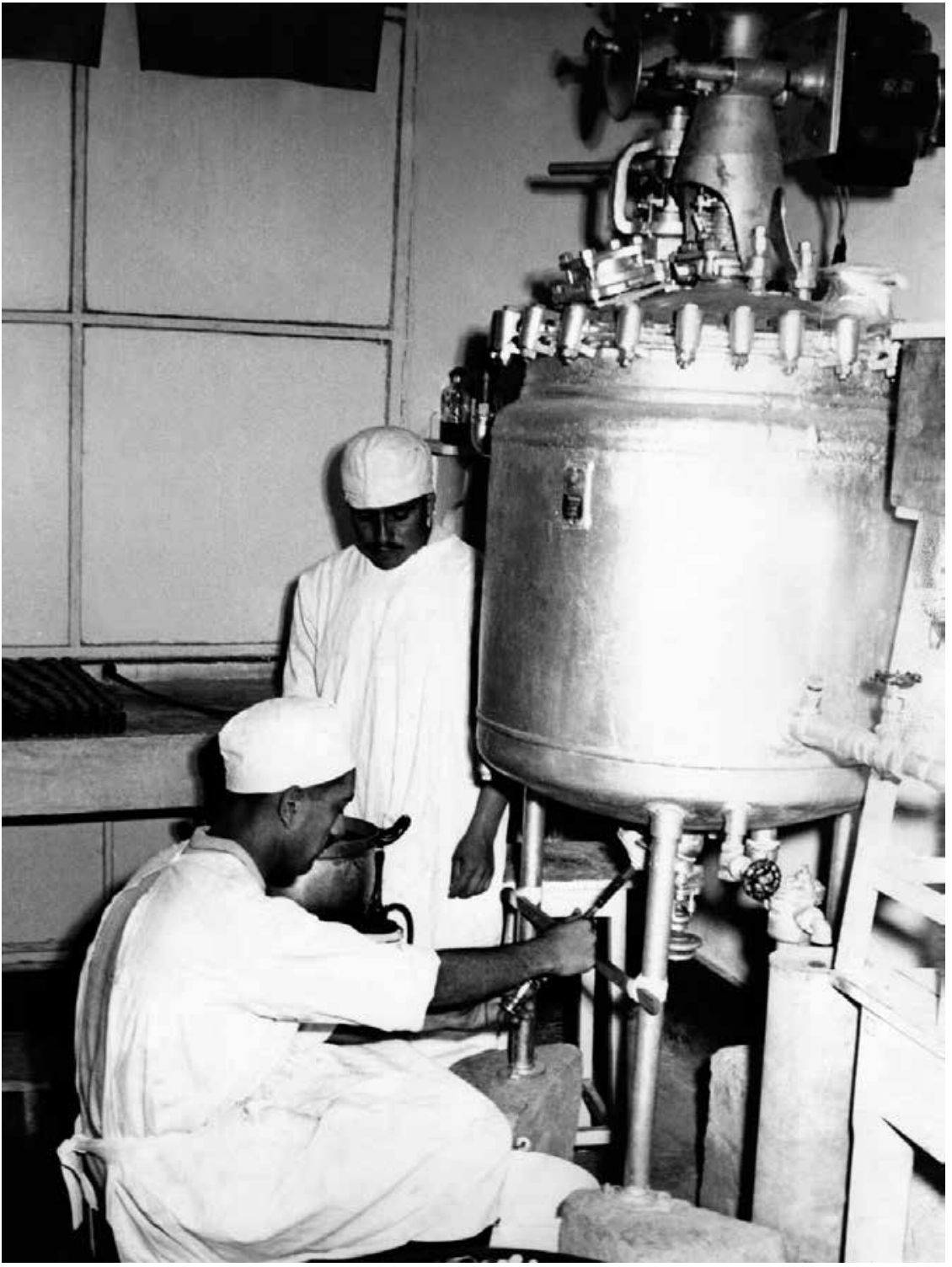

Figura 1. Laboratorio de producción de vacuna antivariólica del Instituto Nacional de Higiene y Salud Pública, 1955 - 1960 (Instituto Nacional de Salud desde 1957). Fuente: Instituto Nacional de Salud.

ministerios de salud y ofrecían la totalidad de los servicios públicos en las áreas bajo su responsabilidad. La División de Servicios Profesionales estaba organizada para apoyar las unidades operativas del SCISP y a la vez prestaban servicios para los programas nacionales del ministerio de salud. Estos servicios incluían medicina, ingeniería sanitaria, enfermería, servicios sociales, veterinaria en salud pública, estadística, planeamiento y educación sanitaria. La división de programas especiales, agrupaba a unidades para el desarrollo de encargos o proyectos específicos del ministerio que operaban como unidades independientes. El organigrama del SCISP para el año 1954 se observa en la Figura 2.

EI SCISP estaba dirigido por un director técnico y un gerente administrativo de origen norteamericano. Entre 1942 y 1961 tuvo seis directores. El primero fue Major Westfall (1942-1944), seguido por Burke Howard (1944-1946), Fred Wampler (1946-1951), F. Harley Paul (1951-1953), Fred Vintinner (1953-1959) y Charles Williams (1959-1962). Desde el inicio, todas las unidades de salud estaban a cargo de personal peruano. En el año 1958, el SCISP tenía un staff de 17 técnicos norteamericanos y tenía con- tratados a 711 empleados peruanos. De ellos, 370 eran trabajadores y técnicos especializados, 179 administrativos y 162 trabajadores de apoyo. Respecto al financiamiento, los términos del convenio planteaban un financiamiento conjunto de ambos gobiernos en las acciones del programa, a la par de la transferencia de fondos nacionales para operaciones por encargo. Para el periodo 1942-1961, se invirtió en el programa un total de 17290 346 dólares de los cuales los Estados Unidos contribuyó en un 23\% ${ }^{(24)}$.

\section{AÑOS FINALES DEL SERVICIO COOPERATIVO INTERAMERICANO DE SALUD PÚBLICA EN EL PERÚ: 1960-1962}

Cambios en el contexto sectorial: 1957-1962

Al iniciarse el segundo gobierno de Manuel Prado, en julio de 1957, se estaba muy lejos de haber alcanzado los objetivos de reorganización del MSPAS para la racionalización administrativa de sus niveles central y periféricos. Las actividades de las estructuras encargadas de la conducción técnico-normativa de los programas preventivos se mantenían separadas de las encargadas de los programas asistenciales, no habiendo entre ellas ninguna relación, inclusive había emulaciones y celos por los ámbitos y recursos asignados, conductas que promovían la ineficiencia. Según Julio Muñoz Puglisevich: "Era un ministerio desintegrado. Desde Lima se gobernaban todos los servicios de salud del país. Yo era Jefe del Departamento de Higiene Escolar y Educación Sanitaria y tenía personal que dependía de nuestro departamento en Cuzco, Puno, La Libertad, etc... El personal de los distintos departamentos técnicos ubicados en la misma localidad no se conocían entre ellos... eran totalmente independientes..." (25).

Con la intención de corregir esa situación administrativa, el ministro Jorge Haaker Fort nombró, el 14 de enero de 1957, una Comisión Encargada de Estudiar la Reestructuración del MSPAS. Entre sus miembros se encontraban los doctores Alfredo Lynch Cordero, Julio Muñoz Puglisevich, Oswaldo José Da Silva y 
Tabla 1. Principales becados del Instituto de Asuntos Interamericanos (IAI) según profesión y especialidad en salud pública, $1942-1960$.

\section{Nombres y Apellidos}

1 - Acha Jamet, Pedro **

2 Alfaro Álvarez, Carlos **

3 Alvarado de Malpartida, Delia

4 Aranguri Lara, Marcial

5 Atkins Morales, Jorge **

6 Bonifacio de Vázquez, Elsa

7 Botto Vargas, Víctor

8 Cano Gironda, Luis

9 Carlin Ochoa, César

10 Collazos Chiriboga, Carlos

11 Díaz Lira, Guillermo

12 Fiek Campodónico, Otto

13 García Erazo, Antonio **

14 Gayoso Pantoja, Carlos **

15 Gonzáles Domeeg, Carlos

16 Gordillo Zuleta, César

17 Lari Cavagnaro, Alberto **

18 León Ugarte, Mario **

19 Macher Becerra, César **

20 Mártinez Bracamonte, Nicolas

21 Muñoz Puglisevich, Julio **

22 Petrozzi Gratta, Armando

23 Ponce de León Alarcón, Juan **

24 Quiroz Salinas, Carlos **

25 Reategui Weninger, Ignacio

26 Salinas Cáceres, Jorge

27 Seretti, Enrique

28 Sotelo Basselli, Alejandro **

29 Temoche Benites, Abelardo **

30 Vallejo Espinoza, Nilo **

31 Vargas Arenas, Raúl **

32 Villa Crespo, Manuel **

33 Villalobos Carranza, Enrique

34 Zapatel Pimentel, Juan **

35 Zavala Cavassa, Alfonso

Profesión

Médico veterinario

Médico

Enfermera

Estadístico

Médico

Enfermera

Médico

Médico

Médico

Médico

Médico

Médico

Médico

Médico

Odontólogo

Médico

Médico

Médico

Ingeniero sanitario

Médico

Médico

Médico

Médico

Médico

Médico

Ingeniero

Médico veterinario

Médico

Medico

Médico

Demógrafo

Médico

$$
\text { Médico }
$$

Médico veterinario

Ingeniero sanitario

** Expertos peruanos que prestaron servicios de funcionarios o asesores de la OPS/OMS.

Abraham Drobny. Estos dos últimos, jefes de misión de la OPS y del IAI, respectivamente. Visto el informe de la comisión, se aprobó el Decreto Supremo de 14 de junio de 1957, que se publicó cuatro días después. Decreto en el que vuelve a insistir en la centralización técnica y la desconcentración administrativa del ministe- rio, así como se formaliza la integración de sus servicios preventivos y asistenciales tanto a nivel central como local, con tendencia a la desaparición definitiva de los servicios o programas verticales ${ }^{(22)}$.

Es así que en el año 1957 se instituyó una nueva y poderosa Dirección General

\section{Especialidad}

Salud pública veterinaria

Educación para la salud

Enfermería de salud pública

Bioestadística

Administración de salud

Enfermería de salud pública

Administración de salud

Control de la tuberculosis

Salud ocupacional

Nutrición

Educación para la salud

Protección materno infantil

Administración de hospitales

Control de enfermedades venéreas

Odontología sanitaria

Administración de Salud

Salud internacional

Capacitación en salud pública

Saneamiento del ambiente

Administración de salud

Higiene y salud escolar

Planificación en salud

Salud internacional

Administración de salud

Administración de salud

Planificación en salud

Salud pública veterinaria

Planificación en salud

Estadísticas en salud

Educación para la salud

$$
\text { Bioestadística }
$$

Administración de salud

Control de la malaria

Salud pública veterinaria

Saneamiento del ambiente de Salud, como única instancia política-administrativa entre el Despacho Ministerial y las otras direcciones del ministerio; con la intención de avanzar de una etapa de integración de servicios preventivos en el ámbito central y local, aún no concluida, a otra etapa de integración de servicios preventivos y asistenciales en el ámbito local, 


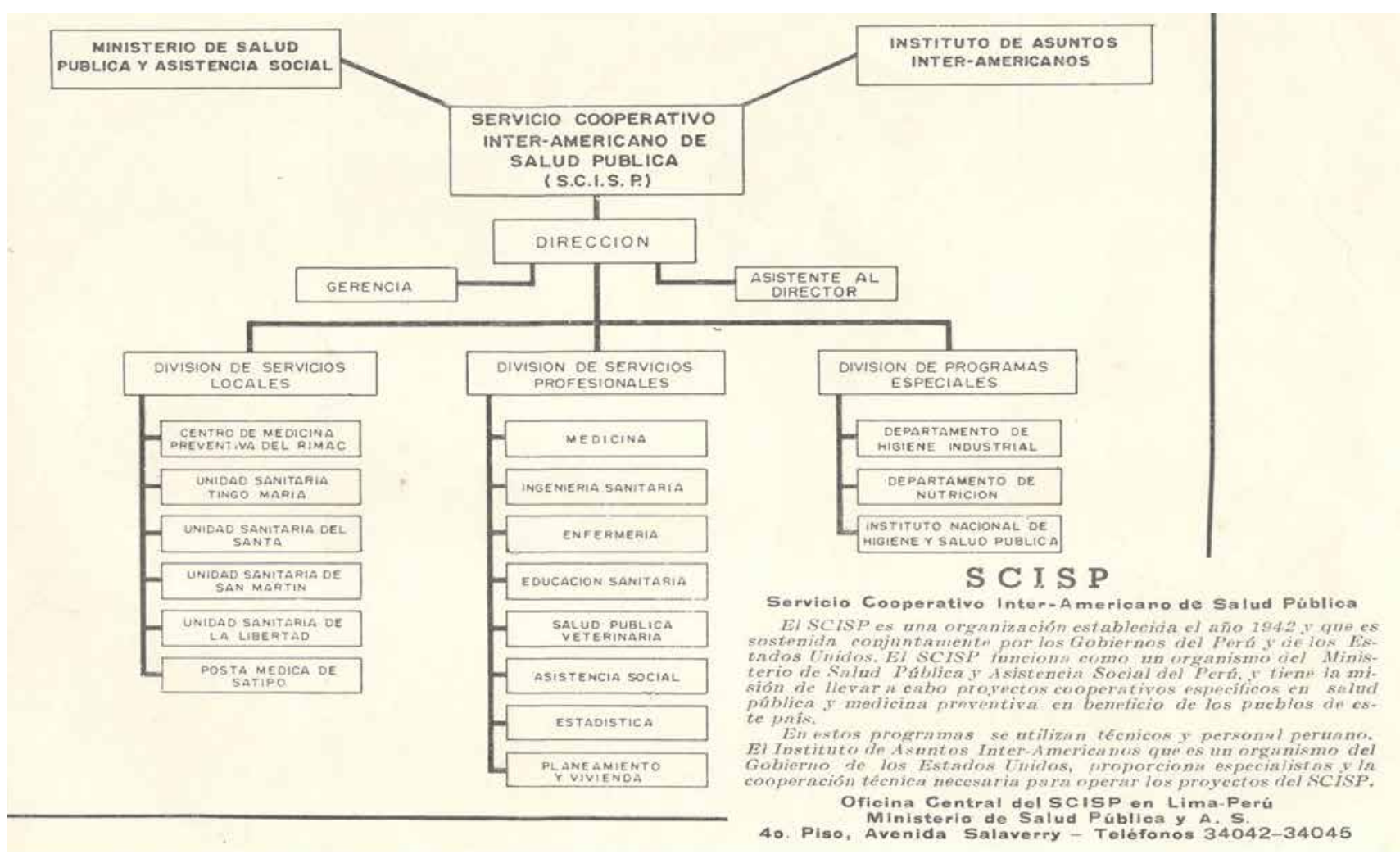

Figura 2. Organigrama del Servicio Cooperativo Interamericano de Salud Pública en el Perú, 1954

bajo el régimen de una sola autoridad política nacional. Se pretendía avanzar y fortalecer la aplicación del principio de la integralidad en la organización y en la dinámica del ministerio, haciendo desaparecer la indeseable y artificial división entre lo preventivo y lo asistencial ${ }^{(22)}$.

Nueva etapa del Servicio Cooperativo Interamericano de Salud pública en el Perú: 1960-1962

El convenio básico que gobernaba las operaciones del SCISP en el Perú expiró el 30 de junio de 1960. Extensiones provisionales permitieron seguir participando formalmente al SCISP en programas especiales en el país hasta mediados de 1961. Estos programas serían asumidos, posteriormente por el Servicio Especial de Salud Pública (SESP), creado por Ley 13908, del 25 de enero de $1962^{\text {(26). }}$

Durante este lapso, el director del SCISP fue el Dr. Charles L. Williams Jr. Conforme a lo previsto en el convenio, se nombró por primera vez un técnico peruano en salud pública como Director Asociado del SCISP. Este técnico, nombrado el 1 으 de noviembre de 1960 , fue el Dr. Jorge Atkins Morales, en esos días Director General de Salud del MSPAS y quien anteriormente había formado parte del grupo de técnicos del SCISP de 1944 a 1954 como jefe de los programas en Loreto ${ }^{(13)}$.

Hasta 1959, los programas del SCISP habían operado de manera paralela a los programas del ministerio, con el propósito de facilitar la organización y tecnificación de determinados procesos. El año de 1960 fue el de transición a una nueva modalidad de trabajo en el SCISP. Su personal dejó de ejecutar programas paralelos al del ministerio en el nivel periférico o en la organización y tecnificación de procesos, y se trasladó al nivel central a trabajar junto con las autoridades sectoriales nacionales, cooperando en la programación y en la ejecución de programas que requerían autonomía en su acción para el mejor cumplimiento de sus objetivos. El 30 de junio de 1960, los servicios u organismos que el SCISP había creado o reorganizado y que aún seguían bajo su gestión en el nivel periférico $u$ operativo, comenzaron a pasar a la administración inmediata de las áreas y unidades de salud del MSPAS ${ }^{(13)}$.

En los siguientes meses, el SCISP desarrolló - con esta nueva modalidad de trabajo - varios importantes programas en el marco de las nuevas políticas sanitarias de fortalecimiento de la desconcentración administrativa y de la integración técnica de los servicios del cuidado de la salud. Entre ellos, los que a continuación se resumen:

- Fortalecimiento de las dependencias de la Dirección General de Salud del Ministerio de Salud Pública incorporadas y administradas por el SCISP. Incluyen al Instituto de Nutrición, Instituto de Salud Ocupacional y el Instituto Nacional de Salud.

- Programa Nacional de Educación Sanitaria. Conforme al acuerdo de noviembre de 1959, de ayuda a la División de Educación para la Salud en la organización del Programa Nacional. Se inicia la formación en el país de educadores de salud pública. 
- Programa № 3: Plan Nacional de Salud; organización de la elaboración de un Plan de Salud Pública con ayuda internacional. Con una programación de tres años.

- Programa № 6: Bioestadística. EI SCISP recolectaba y procesaba las estadísticas vitales y de salud del Perú.

- Programa de Capacitación de Personal; se creó el año 1961 por un acuerdo con el MSPAS y el director del SCISP. Autorizó para el año 1962 una partida para iniciar el desarrollo del programa.

- Ingeniería Sanitaria. En agosto de 1960, conforme al convenio suscrito entre el MSPAS y el SCISP se integraron en una sola división las estructuras de ingeniería sanitaria de ambas entidades. La división seguía siendo una dependencia técnica de la Dirección de Servicios Técnicos-Normativos del MSPAS, pero administrada por el SCISP. El fin de esta integración era potenciar el desarrollo del Programa Nacional de Ingeniería Sanitaria del MSPAS.

- Programa de ayuda técnica a los servicios de salud de las localidades del país. En 1960 se creó un sistema que permitiera proporcionar ayuda técnica y económica, en materiales y equipo, así como facilitar la supervisión de parte de técnicos del ministerio y del SCISP a los servicios periféricos de salud. Ayuda técnica en las áreas de: administración sanitaria, enfermería, servicio social, medicina veterinaria, arquitectura, mantenimiento.

- Programa № 106: Inventario y Estudio Nacional para el Desarrollo de Agua Potable en Ciudades y Pueblos de la República. En 1961 se concretó un acuerdo multinstitucional para el estudio y la formulación de ante-proyectos para la instalación de sistema de abastecimientos de agua potable en localidades con una población superior 500 habitantes. Acuerdo financiado por IAI, SISP, y los fondos nacionales de desarrollo económico y de salud y bienestar social del SCISP. El número de esas localidades era estimado en mil (13).

Aquello ya era posible y razonable debido a que, después de 18 años del inicio de las operaciones del SCISP, la situación técnico-administrativa del ministerio había cambiado. Gracias a los programas de capacitación y entrenamiento del personal, el ministerio se había tecnificado y disponía de un buen número de especialistas en salud pública que ya dirigían las divisiones y departamentos ministeriales, así como las áreas y unidades de salud organizadas. Dos años después, finalizada la última ampliación de contrato con el gobierno de Estados Unidos, las funciones del SCISP fueron asumidas formalmente por el Servicio Especial de Salud Pública ${ }^{(24)}$.

\section{DISCUSIÓN}

El contexto de la II Guerra Mundial apareció en el campo de la cooperación técnica y económica sanitaria, una nueva modalidad cooperativa más definida y más explícitamente ligada a los intereses de la política desarrollada por los Estados Unidos en esos años, para la América Latina: el SCISP. Se trataba de fortalecer la influencia política norteamericana, a partir de programas sanitarios, ejercida hasta entonces a través de las recomendaciones y las acciones de la Oficina Sanitaria Panamericana, la Fundación Rockefeller y el Servicio de Salud Pública de Estados Unidos. Los objetivos geoestratégicos de estas acciones, buscaban mantener la lealtad de los países latinoamericanos al bloque occidental en la guerra fría, además de dinamizar las economías locales a partir de las mejoras de las condiciones de vida a fin de abrir nuevos mercados para la industria norteamericana (27).

El despliegue del SCISP en el Perú fue progresivo, empezando por el puerto de Chimbote y por la expansión paulatina de las acciones a la selva peruana. Las dificultades de inicio eran muy grandes, por un lado, las necesidades del país eran inmensas ante la inexistencia de infraestructura sanitaria en las zonas rurales. Los costos de operación, por la ausencia de carreteras y la dispersión poblacional fueron elevados, de hecho, reportes norteamericanos señalaron que, en determinado momento, se vieron obligados a abandonar algunas operaciones sanitarias como la existente en el departamen- to de Madre de Dios por no ser sostenibles ${ }^{(28)}$.

De otro lado en el Perú, existía una gran carencia de recursos humanos, en especial médicos, por lo que el SCISP se vio obligado a contratar estudiantes recién egresados con una serie de incentivos. Los testimonios de los funcionarios peruanos que trabajaron en el SCISP coinciden en destacar las satisfactorias condiciones de salario, trabajo y desarrollo profesional que encontraron en el servicio; condiciones muy superiores a las existentes en otras dependencias sanitarias del país y qué en su entender, garantizaban la adquisición de las competencias requeridas para el cumplimiento de los compromisos institucionales con la salud poblacional. Por otra parte, diversos autores coinciden en señalar que el SCISP hizo importantes aportes a la tecnificación y modernización de la salud pública nacional; aunque, también coinciden en comentar que los efectos prácticos de esos aportes no pudieron ser mantenidos a mediano plazo, ni mucho menos generalizados al resto del país, debido a que se vieron afectados seriamente por un error de enfoque, cometido por la IAI en sus 20 años de operación en América Latina. Error que consistió en pretender estandarizar un esquema técnico, administrativo y económico-financiero para toda América Latina, sin considerar las diferentes circunstancias y capacidades nacionales para aplicar y sostener tal esquema (22), tal como lo señaló Cueto: "Los médicos de salud pública formados en los Estados Unidos fueron muchas veces socializados para trabajar en un medio que no tenía las carencias del peruano. Por ello, muchas veces su entrenamiento acabó siendo un fenómeno aislado..." (3).

La revisión crítica de la historia del SCISP que hemos efectuado nos conduce inevitablemente a evaluar el impacto de sus acciones en nuestro sistema de salud. En términos generales el Servicio Colaborativo Interamericano en Salud Pública fue una notable inyección de recursos y transferencia tecnológica en salud en el país. La introducción de servicios de salud en diversos puntos de la selva peruana, los avances importantes de las campañas de saneamiento, la erradicación y control de enfermedades transmitidas 
por vectores, la transferencia de capacidades y tecnologías que permitieron, entre otros logros, la fabricación local de vacunas, constituyeron hitos notables en el desarrollo del sistema de salud peruano que no hubieran sido posibles sin el financiamiento y la transferencia tecnológica de la cooperación norteamericana (29). Es importante señalar, la importante contribución de algunos funcionarios peruanos como Carlos Quirós, que lograron desde las direcciones técnicas del Ministerio de Salud de aprovechar los éxitos logrados por el SCISP para obtener mayores fondos del Estado Peruano para el control de enfermedades y reorientar de forma eficiente la acción de los recursos disponibles para el logro de importantes objetivos sanitarios del país ${ }^{(30)}$.

Consideramos que, a pesar de sus errores de enfoque e intereses institucionales, el SCISP tuvo una influencia importante en el desarrollo de la salud pública nacional, a través de la actuación del personal que adiestró y que formó dotándolo de una especial mística de trabajo. Este personal profesional conformó en las décadas del 50 y del 60, el núcleo de una generación de sanitaristas peruanos que, con una calidad y un compromiso admirables, lideró e impulsó un importante desarrollo tecnológico y material de las instituciones sanitarias y de seguridad social. Un número importante de los sanitaristas ex-becarios del IAI fueron captados, por sus altos méritos, como funcionarios o asesores de la Organización Panamericana de la Salud, pero además se constituyeron en una comunidad epistémica: la generación de los "sanitaristas" que dejaron un importante legado de políticas, que influyo notablemente en el Ministerio de Salud un par de décadas después del término de las acciones del SCISP y que marco todo un horizonte de desarrollo institucional.

Varios elementos explican la falta de sostenibilidad de muchas de las intervenciones y capacidades desarrolladas por el SCISP en el Perú, una de ellas fue la propia matriz cultural de la salud pública oficial latinoamericana que fue surgiendo en el contexto de cooperación norteamericana durante la Guerra Fría, lo que varios autores denominan, "cultura de la sobrevivencia" y que se caracterizó én- fasis exagerado en las tecnologías médicas, fragmentación del sistema de salud, discontinuidad de las políticas sanitarias y promoción de un concepto de la salud pública como una respuesta temporal a las emergencias ${ }^{(31)}$. Un elemento concurrente a esta cultura de la supervivencia fue el contexto político peruano, el cual estuvo hegemonizado durante el periodo más importante de la actividad del SCISP, por el gobierno de Odría, que desplegó un populismo que impregnó las acciones de asistencia social (32). La "cultura de la sobrevivencia", era muy funcional al pragmatismo de un gobierno como el de Odría; totalmente alineado con los intereses norteamericanos y que por lo tanto, no tenía incentivos para negociar políticamente la posibilidad de desarrollar algunas iniciativas locales que Ilevaran a la cooperación norteamericana a acciones orientadas a fortalecer una agenda política local, cosa distinta a lo ocurrido en Bolivia, donde el gobierno de Paz Estensoro negoció con el gobierno de los Estados Unidos, a partir del SCISP, la implementación de programas de salud orientados al mundo rural ${ }^{(33,34)}$.

Es sumamente interesante el hecho, que desde el inicio de la concepción del Servicio Cooperativo Interamericano en Salud Pública, como un programa de asistencia para toda América Latina, se haya concebido el desarrollo de hospitales o dispensarios locales, proyectados para la acción en territorios específicos. En 1944 las acciones de construcción y rehabilitación de hospitales abarcaban a 10 países de la región y comprendían aproximadamente la construcción o rehabilitación de 100 hospitales. Se desarrollaron estos servicios como centro de operaciones para un conjunto de intervenciones de salud pública en el territorio (inmunizaciones, programas materno infantiles, control de enfermedades venéreas, etc.), a las cuales se sumaron diversas acciones como programas de saneamiento local e intervenciones extramurales de educación sanitaria ${ }^{(35)}$, que incluían el uso del cine. La elección de las zonas de construcción u operación de estos hospitales (en algunos casos el programa incluía la rehabilitación y equipamiento de hospitales ya existentes), generalmente, estaba en relación con el control de epide- mias y la mejora de condiciones de salud en territorios donde los norteamericanos tenían intereses específicos, sobre todo de asegurar el acceso a materias primas estratégicas. En su concepción inicial, se previó que estas unidades, tuvieran un efecto demostrativo de la eficacia de la salud pública moderna a fin de transferir posteriormente a los gobiernos de los países beneficiarios de la cooperación su operación y financiamiento de dichos servicios.

El reconocimiento de esa influencia positiva del SCISP no implica, ciertamente, negar que los beneficios de dicho desarrollo solo alcanzaban a un porcentaje limitado de la población peruana, en tanto obedecía, finalmente, a una política nacional de desarrollo general de carácter populista-oligárquico, en un contexto regional de dominio de las ideas tecnocráticas de la salud pública estadounidense. Las autoridades peruanas nunca se plantearon un proyecto de desarrollo institucional estatal que permitiera sostener en el largo plazo los desarrollos y capacidades generadas por el SCISP y en el caso de los hospitales, centros de medicina preventiva y dispensarios territoriales, no se logró desplegar su expansión en el conjunto del territorio. En la década de los sesenta lograron mantenerse muchas intervenciones desarrolladas, a la par que se alcanzó mayor financiamiento para el Ministerio de Salud, lo que permitió el inicio de desarrollos en planificación orientados al desarrollo de un mayor despliegue territorial del sistema de salud ${ }^{(41)}$. Sin embargo, la falta de continuidad en los procesos de diseño y reforma del incipiente sistema de salud peruano derivado de los cambios en los procesos políticos en la segunda mitad de la década del 70, asociados a una severa caída en el financiamiento al final de dicho periodo, significó un deterioro progresivo de las capacidades acumuladas gracias a la cooperación del SCISP.

Sin embargo, consideramos que existen un conjunto de legados y prácticas institucionales derivadas de la experiencia del SCISP que lograron mantenerse a lo largo del desarrollo posterior del Ministerio de Salud y algunas continúan hasta hoy, como por ejemplo el autoritarismo en la implementación de algunos 
programas verticales ${ }^{(36)}$, la tendencia fragmentación de la organización de las intervenciones sanitarias, el respeto a la autoridad de los tecnócratas-expertos y la excesiva autonomía y poder de los mismos ${ }^{(37)}$, que no se sienten obligados a consultar a las poblaciones beneficiarias de las intervenciones o de las políticas y sobre todo el hecho que, ante la necesidad del ministerio de dar saltos cualitativos en su desarrollo técnico-institucional, la proclividad de encargar a entes externos, el diseño e implementación de capacidades que después son difíciles de institucionalizar ${ }^{(38)}$.

Es importante señalar que nuestro estudio es un ejemplo de lo que Dargent denomina "determinantes internacionales de la capacidad de las organizaciones del estado" (39). Nuestros hallazgos plantean que la construcción del sistema de salud peruano, estuvo fuertemente ligada a los intereses geopolíticos de los Estados Unidos en el contexto de la guerra fría, siendo las acciones del SCISP fundamentales para entender los patrones de construcción de la matriz institucional del sector salud, donde se expresa con particular vigencia en la actualidad, la continuidad de una "cultura de sobrevivencia"; la cual es un serio obstáculo para el desarrollo del sector salud peruano, de cara a los complejos retos que nos plantea el actual escenario global del siglo XXI.

\section{REFERENCIAS BIBLIOGRÁFICAS}

1. Guzmán M del P, Quevedo E. North American Technical Cooperation in Public Health Policy in Colombia During the Second World War. Biomédica.1999;19(1):5-17.

2. Garcia J. La medicina estatal en América Latina. En: Pensamiento social en salud en América Latina. México: Interamericana McGraw-Hill, OPS; 1994. p. 95-143.

3. Cueto M. Una Reforma Fallida: la Fundación Rockefeller, la Malaria y la Salud Pública en el Perú. Revista Peruana de Ciencias Sociales. 1990;2(2):9-29.

4. Eslava JC. North American influence on the development of public health in Colombia. Biomédica. 1998;18(2):101-9.

5. Conferencia Sanitaria Panamericana. Acta final de la Undécima Conferencia Sanitaria. [Internet]. Rio de Janeiro; 1942 18/9 [citado el 24 de octubre de 2019]. Disponible en: http://iris.paho.org/xmlui/ handle/123456789/28735
6. Carvallo C. Servicio Cooperativo Interamericano de Salud Pública. En: Memoria del Ministerio de Salud Pública y Asistencia Social, julio 1941-julio1945. Lima: Imprenta Torres Aguirre S.A; 1945. p. 277-91.

7. Villacrespo M. Testimonios de Personajes de la Salud Pública Peruana. Entrevista por Manuel Campos Sánchez el dia 13 de agosto de 1984. Escuela de Salud Pública del Perú; 1985.

8. Westphal E. Servicio Cooperativo Interamericano de Salud Pública. Boletín de la Dirección General de Salubridad. Lima: Imprenta Minerva; 1943. 91-96 p.

9. Vallejos N. Testimonios de Personajes de la Salud Pública Peruana. Entrevista efectuada por Manuel Campos Sánchez, el dia 20 octubre de 1984. Escuela de Salud Pública del Perú; 1985.

10. Ministerio de Salud Pública y Asistencia Social. El Servicio Cooperativo Interamericano de Salud Pública: 1942-1960. Lima: Ministerio de Salud Pública y Asistencia Social; 1962.

11. Lynch Cordero A. Entrevista al Dr. Alfredo Lynch Cordero, efectuada por el Dr. J. R. Cornejo Ubillús el 24 de febrero de 1996 (informe inédito). En: Registro BiográficoVivencial (Vivigrama) de la Historia de la Salud Pública Perú: 1956-1999. Lima: Programa de Fortalecimiento de Servicios de Salud, Ministerio de Salud; 1998.

12. Quiroz C. El Hospital de La Caleta de Chimbote. Revista Peruana de Epidemiología. 1995;8(1):57-60.

13. Ministerio de Salud Pública y Asistencia Social. Informe de actividades y programas del Servicio Cooperativo Interamericano de Salud Pública. Lima: Ministerio de Salud Pública y Asistencia Social; 1960.

14. Westphal E. Discurso con ocasión de la inauguración del Centro de Medicina Preventiva del Rímac. La Reforma Médica. 1945;(456):505.

15. Servicio Cooperativo Interamericano de Salud Pública. El Centro de Medicina Preventiva del Rimac en su $10^{\circ}$ aniversario. Lima: SCISP y MSPAS; 1955.

16. Reategui R. Testimonios de Personajes de la Salud Pública Peruana. Entrevista efectuada por Manuel Campos Sánchez, el día 20 julio 1984. Escuela de Salud Pública del Perú; 1985.

17. Ferrandiz J. Alberto Hurtado y las Enfermedades Ocupacionales. Acta Herediana. abril de 2000; Volumen Conmemorativo 28/29:35-8.

18. Estrella Ruiz J. Prontuario de la Legislación Sanitaria del Perú Tomo IV. (Primera y segunda parte). Lima: Librería e Imprenta D. Miranda; 1947.

19. División de Higiene y Educación Sanitaria. Informe del Departamento de Nutrición. Salud y Bienestar Social. Salud y Bienestar Social. 1955;4(10-11):118-20.

20. Collazos C, Moscoso I, Bravo de Rueda Y, Castellanos A, Cáceres C, Roca A, et al. La alimentación y el estado de nutrición en el Perú. Anales de la Facultad de Medicina. 1960;43(1):7-357. DOI: https://doi. org/10.15381/anales.v43i1.6288

21. USAID. Una historia de 70 años de colaboración en salud con el Perú. 2a ed. Baltimore: Johns Hopkings University; 2015.

22. Bustios C. La Salud Pública, la Seguridad Social y el Perú Demoliberal (1933-1968). Facultad de Medicina UNMSM-CONCYTEC; 2005

23. Ministerio de Salud Pública y Asistencia Social. Seminario Nacional de Educación Sanitaria. Lima; 1954.
24. Morris P. History of Technical Assistance in Perú 19421962 [Internet]. Disponible en: https://pdf.usaid.gov/ pdf_docs/PNADZ142.pdf

25. Muñoz Puglisevich J. Entrevista al Dr. Muñoz P., efectuada por el Dr. J. R. Cornejo Ubillús el 24 de febrero de 1996 (informe inédito). En: Registro Biográfico-Vivencial (Vivigrama) de la Historia de la Salud Pública Perú: 1956-1999. Lima: Programa de Fortalecimiento de Servicios de Salud, Ministerio de Salud; 1998.

26. Servicio Especial de Salud Pública. Informe Anual de Actividades del Servicio Especial de Salud Pública 1963. Lima: Imprenta SESP; 1963.

27. Cueto M, Brown T, Fee E. The Word Health Organization. A History. Ginebra: Cambridge University Press; 2019. 86-90 p.

28. Jackson D. Special study misión to Latin America on tecnical cooperation. Committee on Foreign Affairs. H.Res.113. Washington; 1954. 48-50 p.

29. Griffing SM, Gamboa D, Udhayakumar V. The history of 20th century malaria control in Peru. Malaria Journal. 2013;12(1):303.

30. Quirós S. Memorias de un Salubrista. Política y Salud en el siglo XX. 1a ed. Lima: Universidad Nacional Mayor de San Marcos; 2019. 133-137 p.

31. Cueto M. La "cultura de la sobrevivencia" y la salud pública internacional en América Latina: la Guerra Fría y la erradicación de enfermedades a mediados del siglo XX. História, Ciências, Saúde-Manguinhos. 2015;22(1):255-73.

32. Candela J, Contreras Z, Lossio C. Populismo y Salud Pública durante el Ochenio de Odría (1948-1956). Acta Herediana. 2017;60:33-48.

33. Pacino N. Stimulating a Cooperative Spirit? Public Health and U.S.-Bolivia Relations in the 1950s. Diplomatic History. 2017;41(2):305-35.

34. Pacino N. Bringing the Revolution to the Countryside: Rural Health Programmes as State-Building in Post1952 Bolivia. Bulletin of Latin American Research. 2019;38(1):50-65.

35. Dunham GC. The Coöperative Health Program of the American Republics. Am J Public Health Nations Health. 1944;34(8):817-27.

36. Cueto M. La Salud Internacional y la Guerra Fría. Erradicación de la Malaria en México, 1956-1971. Universidad Nacional Autónoma de México, Instituto de Investigaciones Históricas; 2013. 147-149 p.

37. Ewig C. Neoliberalismo de la segunda ola. Género, raza y reforma del sector salud en el Perú. 1a ed. Lima: Instituto de Estudios Peruanos; 2012. 165-166 p.

38. Yamin A. Castillos de Arena en el camino hacia la modernidad. Una perspectiva desde los derechos humanos sobre el proceso de reforma del sector salud en el Perú (1990-2000) y sus implicancias en la muerte materna. Lima: Centro de la Mujer Flora Tristán; 2003. 131-132 p.

39. Dargent Bocanegra E. International Determinants of State Agencies' Capacity: Lessons from Colombia and Peru. Apuntes Revista de ciencias sociales. 2014;41(74):9-40.

40. Quirós SC. La Viruela en el Perú y su erradicación. Recuento Histórico. Revista Peruana de Epidemiologia, 1996, 9(1):41-53.

41. Situación de salud del Perú: Exposición del Teniente General F.A.P. Humberto Campodónico Hoyos. Ministro de Salud en el CAEM Abril, 1977. Lima, Perú: Ministerio de Salud;1977. 